\title{
Two-dimensional filtering, oriented line detectors, and figural aspects as determinants of visual illusions
}

\author{
E. G. J. EIJKMAN, H. J. JONGSMA, and J. VINCENT \\ University of Nijmegen, Nijmegen, The Netherlands
}

\begin{abstract}
Quantitative data of Müller-Lyer illusions from the literature were analyzed according to three different models. All three models predict the illusion effect, although with different magnitude and different parameter dependency. First, a filter model describing a certain amount of blurring of the retinal picture seems partly responsible for the observed illusion. With reasonable estimation of the filter constants, however, a sufficient magnitude of illusion cannot be obtained. A second model of oriented line or bar receptors is even less effective in explaining the observed length illusions. A third model, consisting of a size-constancy operator triggered by depth cues, may predict effects larger than actually observed. It is concluded that figural aspects such as depth-inducing cues are mainly responsible for the illusion effects observed in Müller-Lyer figures.
\end{abstract}

Visual geometrical illusions have been studied extensively since the end of the 19th century (e.g., Müller-Lyer (1896) because it was expected that the observed distortions in the visual field would elucidate fundamental properties of visual processing. The results, however inspiring, have not always pointed toward an explaining theory. There does not seem to emerge a single clear theory which would explain all phenomena adequately. Gregory (1970), for instance, advocates a figural explanation by observing that such clear patterns usually elicit depth perceptions and therefore operate on size-constancy mechanisms (Day, 1972; Gauld, 1975; Lester, 1977; Smith, 1978; Ward, Porac, Coren, \& Gingus, 1977). In such depth-producing figures, the occurrence of distortions of length would be plausible. As a noncognitive antipode, the suggestion has been made by Kawabata (1976), among others, that illusions are merely the result of filter processes caused by neural lateral interactions, which he describes by means of a "spatial coupling function." Others (e.g., Caelli, 1977) suggest that oriented line detectors may play a role. There seems to be more than one mechanism contributing to the observed effects so that simple, straightforward explanations are questionable (Pressey \& DiLollo, 1978; Restle \& Decker, 1977). In such cases, purely quantitative theories are called for, in order to decide whether a certain mechanism shows sufficient explicative power.

The present study compares the quantitative effect of three different models for the distortion of length in Müller-Lyer illusions. The first is concerned with the mapping of visual patterns transported along the optical nerve, by which a filtered version of the original pattern is produced. The second model de- scribes the influence of oriented line or bar detectors in the visual cortex. And the third is a simple model of the size-constancy operator.

Experimental data in the literature show a rather consistent picture of the magnitude of distortion of length in Müller-Lyer figures in the most effective range of stimulus parameters. For this reason, we will restrict ourselves to the Müller-Lyer patterns in this range and we will use data accumulated by Davies and Spencer (1977) and Restle and Decker (1977).

All three models, which we will investigate, predict illusions of the kind commonly observed. However, they predict different relations with stimulus parameters. Furthermore, we will be able to predict absolute values of the magnitude of the illusion, thereby acquiring an estimate of the relative importance of the different mechanisms. The interesting point is that physiological data are sufficiently precise for the conclusion that the nervous structures behind the retina and the imperfectly tuned bar receptors of the cortex fail to bring about illusions that match the observed ones. Therefore, an argument emerges for a more figural explanation of the illusion.

\section{THE LOW-PASS FREQUENCY FILTER}

The nervous pathways of the visual filter transmit a copy of the retinal image which is blurred by two effects. First, the rods and cones have finite dimensions and nonzero mutual distances, factors which are detrimental to the perception of fine details of the image. Second, there are neuronal interactions between neighboring nervous elements which prevent the transmission of precise detail below a certain magnitude. The resolution can be set at about 5-10 min 
of arc, according to physiological data. In some cases, however, the visual system manages to obtain better spatial resolution, even by a factor of 10 , especially in cases in which vernier acuity is required. The central visual system probably uses a spatial interpolating mechanism of some kind which, in case of simple structures, can lead to better accuracy of spatial position (for a discussion, see Barlow, 1979). Nevertheless, unexpected breaking points in the retinal pattern cannot be restored by the same interpolation mechanism. At these points, such as those at the ends of lines, the visual system has to deal with diminished spatial resolution. This can be most adequately expressed in the spatial frequency domain by a low-pass filter. In passing this filter, the visual information loses its high-frequency content and the corresponding two-dimensional pattern becomes blurred. The characteristic of the peripheral visual filter is usually given in the spatial domain as the output of a point light source and is called a point spread function. This function is mostly thought of as an excitatory central region surrounded by an inhibitory region, such as that shown in Figure 1 for one dimension. Kawabata (1976) uses a similar function for calculating "visual potential" functions, and he calls it a spatial coupling function. In the present study, we prefer to call it an impulse response function, because then we will use the terminology of system theory and we can apply the mathematics of frequency analysis most readily.

The effect of a filter on an arbitrary pattern can best be formulated in terms of Fourier analysis. Suppose the visual pattern can be described by the intensity of illumination, $z$, of the retinal $x-y$ plane: $z=$ $f(x, y)$. The output, $g(x, y)$, of the visual filter will then be the convolution:

$$
g(x, y)=\iint f(\xi, \eta) h(x-\xi, y-\eta) d \xi d \eta,
$$

with $h(x, y)$ being the impulse response function. Calculation is facilitated by transposing the above expression into the frequency domain by means of

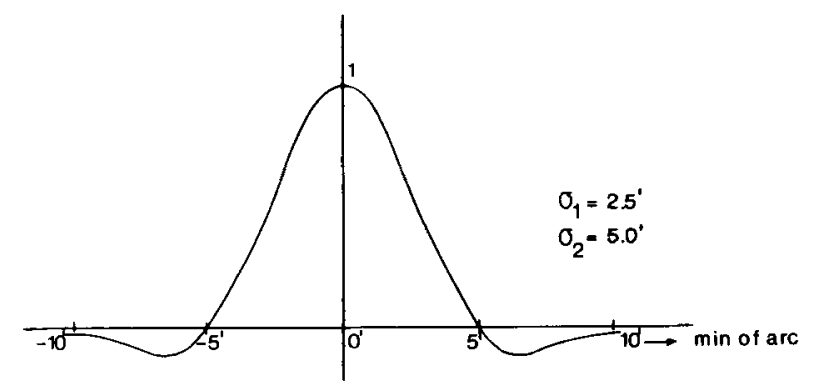

Figure 1. The impulse response function of the peripheral visual system in the plane $y=0$ for a point source at $x=0 ; y=0$. According to psychophysical measurements, the positive phase in the fovea should have a width of $\mathbf{1 0} \mathbf{~ m i n}$ of arc or less.
Fourier integrals and further standard mathematical techniques.

Müller-Lyer patterns have been filtered using the impulse response function

$$
\begin{aligned}
h(x, y)= & (2 \pi \sigma)^{-1}\left[\exp -\left(x^{2}+y^{2}\right) / 2 o^{2}\right. \\
& \left.-1 / 4 \exp -\left(x^{2}+y^{2}\right) / 8 \sigma^{2}\right] .
\end{aligned}
$$

This function corresponds with Figure 1 and is based upon the subtraction of two Gaussian curves $(\sigma=$ $\left.\sigma_{2}=2 \sigma_{1}\right)$. Psychophysical data available on $h(x, y)$ diverge as to the experimental conditions of measurement and show differences of $a$ by a factor of 2 or 3 . Figure 1 shows a representative mean of the fovea (see, e.g., Wilson \& Bergen, 1979), which is also in accordance with electrophysiological evidence.

However, we here encounter a difficult problem in view of the fact that the direction of gaze moves about the pattern during inspection while the resolution of the eye is dependent upon the distance from the central fovea.

Probably, the percept of length is obtained in the first instance by a global inspection of the figure. Both ends of a line are not very well defined at this stage because this global image is rather crude. By eye movements, however, the global image can be made more precise at the sites of foveal fixation. Although it is impossible to retain very many fine details in a complicated picture, we will assume here that both ends of the shaft can be "filled in" with near maximum precision, obtainable with the fovea. This precision can be kept sufficiently long for judgments of length, and therefore the filter that is limiting the observation is the filter of the central fovea and not of the more peripheral parts of the retina.

Figure 2 shows the result of the filtering process. Calculations were carried out in a matrix containing $200 \times 900$ picture points. For purposes of demonstration, the values of $\sigma$ used here is tenfold that of the expected physiological value at reading distance. So Figure 2 represents an exaggerated blurred version of the pattern shown by equal-intensity contours. Maximum filter output is obtained at the site of the two points in Figure 2. The figure as a whole is suggestive of the well-known Muller-Lyer illusion, but more detailed analysis poses a problem. Observers would not confirm that the lines in the MüllerLyer patterns were blurred. As explained earlier, the visual system shows a remarkable potential to restore simple structured patterns, such as lines (Barlow, 1979), but breaking points in these patterns cannot be restored once the high-frequency content at these points is lost. So the lengths of lines are judged with at least an order of magnitude less accuracy than the vernier accuracy for putting two line segments in a straight line. A second problem is posed by the question of which level of output illumination is used for 

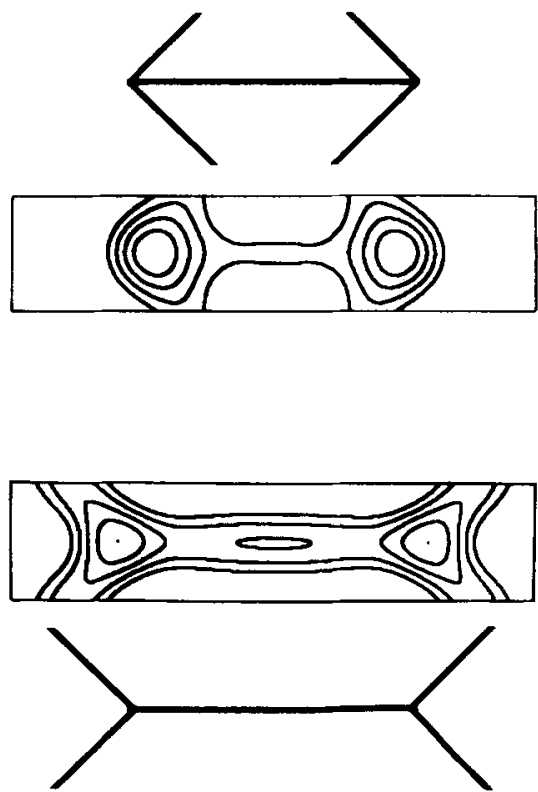

Figure 2. Müller-Lyer patterns with their filtered versions. The blurred pattern has been depicted with equal luminosity lines at $80 \%, 60 \%, 40 \%$, and $20 \%$ of the maximum, which itself is represented as a point. The filter used here has a $\sigma$ that is 10 times the magnitude expected at reading distance. The difference in length of the shaft is apparent in the filtered versions, if corresponding points of intensity are considered.

estimation of line length. In other words: Which characteristic is chosen as an endpoint in a blurred version of the end of a line? The maximum level of excitation is an obvious choice, but there is no guarantee that another intermediate level cannot be used. Fortunately, the choice of level of excitation does not seriously influence the relative magnitude of the illusion in the range of parameters that is used most frequently, so we will proceed with the assumption that it is the maximum that is used for estimation of length.

Filter outputs like the one shown in Figure 2 yield the means to investigate the influence of the angle of the fins and of fin length on the distortion of shaft length. The results are depicted in Figure 3, but now with parameters in the normal physiological range. All lengths are given in visual angles, which is an obvious choice if it is realized that it is the extension of the image on the retina and not the actual length of the figure which determines the filter output. If parameters of the Müller-Lyer patterns are chosen in a commonly used range, that is, a shaft length of $5 \mathrm{~cm}$ viewed at a distance of $35 \mathrm{~cm}$, we cannot expect distortions of length larger than $15 \mathrm{~min}$ of arc corresponding to $3 \%$ of the shaft length.

A comparison of this model with psychophysical data is made below.

\section{ORIENTED LINE OR BAR DETECTORS}

The existence of cortical cells especially sensitive to lines or bars of a particular orientation has been well established. In the last few years, data of monkey cortex have become available (e.g., Schiller, Finlay, \& Volman, 1976), so now we can apply quantitative properties of such cells with more confidence. The tuning of these cells to different orientations of bars ranges from a rather sharp (10 deg) to a broad (120 $\mathrm{deg}$ ) tuning width, with most cells at a width of $40 \mathrm{deg}$. Assume for a moment, now, that at a certain stage in the visual process these cells are carrying all information from which length perception is extracted. The distortion of perceived length of the shaft of a Müller-
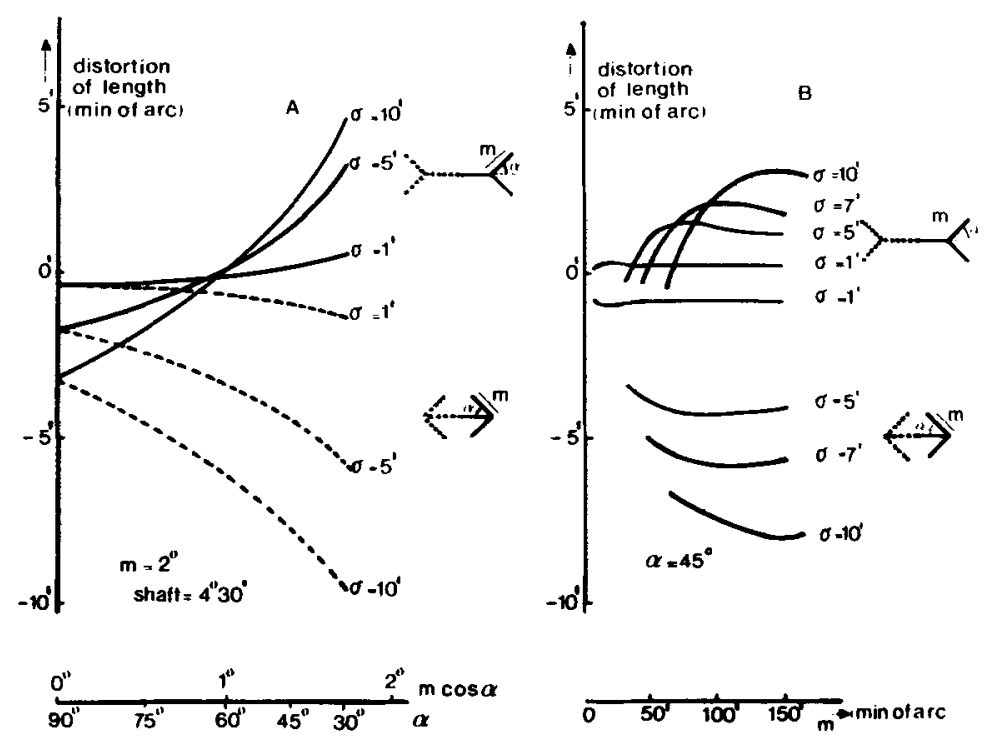

Figure 3. Expected distortion of length calculated with the filter model. In the left graph, fin angle $\alpha$ is varied and filter width $\sigma$ is the parameter. Solid lines represent fins outward; dashed lines represent inward fins. At the right, fin length is varied with $\sigma$ as the parameter. In this figure, shaft length is held constant. 
Lyer pattern can be obtained from this assumption by calculating the contribution of the fins to the response of cells in the direction of the shaft.

It is intuitively apparent that this contribution might influence the apparent length of the shaft. For quantitative modeling of the contribution of all line elements, we will divide the pattern into elementary line segments and calculate the contribution of these elements to the output, I, of a point along the shaft. We will assume the relation

$$
I=\sum_{i} e^{-r_{i} / a} \cos ^{2} \phi_{i},
$$

with $\phi_{i}=$ the angle between the orientations of shaft and line segment, respectively; $r_{i}=$ distance between segment $i$ and considered point along the shaft; $a=$ constant, estimated (from physiological data on receptive field size) to be $10 \mathrm{~min}$ of arc; and $i=$ number of a line segment. The formula describes the imperfectly tuned receptive field of a directionally sensitive neuron with its center on the shaft of the figure. The imperfection causes a contribution from neighboring figure elements at a distance, $r$, weighted by an exponential distance function often used for this purpose. Moreover, the contribution of a neighboring line element is weighted according to the direction of the line relative to the shaft by $\cos ^{2} \phi$ function which seems apropriate to describe electrophysiological results (Schiller et al., 1976) of directional sensitivity.

Contributions to a shaft field come both from neighboring shaft elements and from neighboring fin elements, the latter having a smaller influence because they do not have the proper direction: $\phi \neq 0$.

For a description of the effect, the number of line

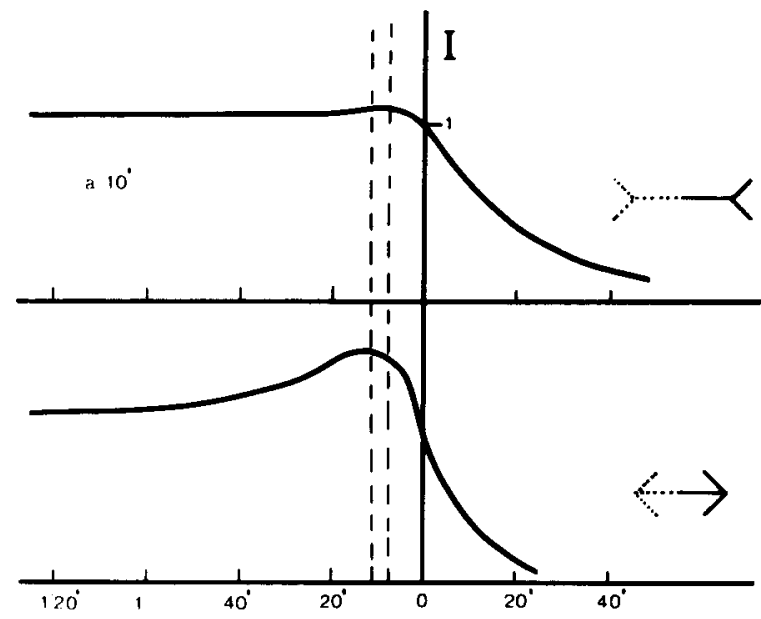

Figure 4. Calculated output of oriented bar detectors along the position of the shaft. There is a small amount of length distortion, depending on which level of output $I$ is used for defining length. Parameters for this calculation are as follows: $\alpha=45$ deg; $a=10 \mathrm{~min}, m>1 \mathrm{deg}$, shaft length $>2 \mathrm{deg}$, and number of elements $i_{\max }=20$.
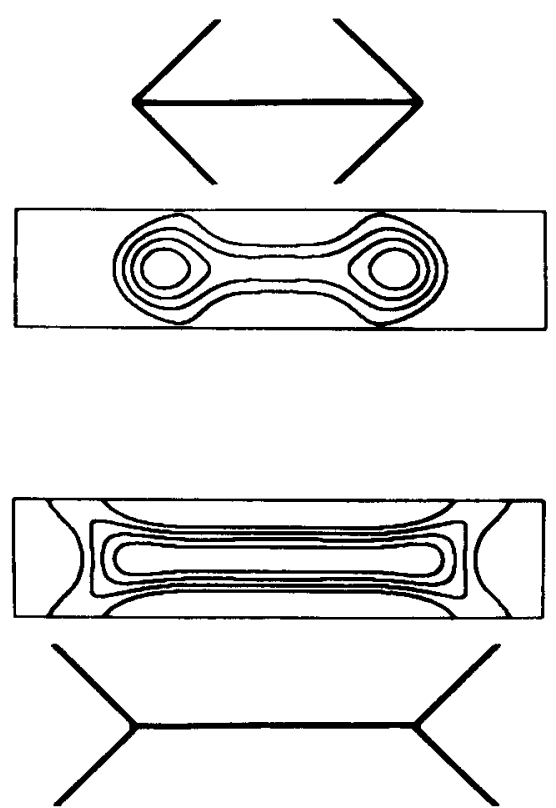

Figure 5. The convoluted effect of filter output and oriented bar detector output. The total effect is less than that obtained in Figure 2. Again, the filter width has been exaggerated for demonstration purposes ( $\sigma=50 \mathrm{~min} ; a=1.7 \mathrm{deg}$ ).

elements, $i_{\max }$, is of little importance once it exceeds 10 elements. The calculation has been extended to points beyond the line elements of the shaft because there is an output to be expected for receptive fields outside the actual length of the shaft. The result, for a particular set of parameters, is shown in Figure 4. Perhaps unexpectedly, fins in and out both produce maxima at distances shorter than the actual length of the shaft. But then a single line would seem shorter too if the same calculation were applied. The difference in length between the two patterns judged from the shift of maximum intensity is about $5 \mathrm{~min}$ of arc. This would correspond with a maximum distortion of length of $1 \%$ of a $5-\mathrm{cm}$ pattern viewed at a distance of $35 \mathrm{~cm}$. If the length was estimated from a much lower intensity level, one could reach a distortion of nearly $3 \%$.

A natural question would be whether the peripheral low-pass filter and the more central oriented-bardetector ought to be combined in order to describe the full illusion effect. In Figure 5, this is shown for a single set of parameters. It appears that the oriented bar receptors contribute very little, if anything, to the already existing effect brought about by the filter output.

\section{THE SIZE CONSTANCY OPERATOR}

In experiments, the Müller-Lyer figures used are most frequently symmetrical, with equal fins. For such instances, the size constancy operator induced 


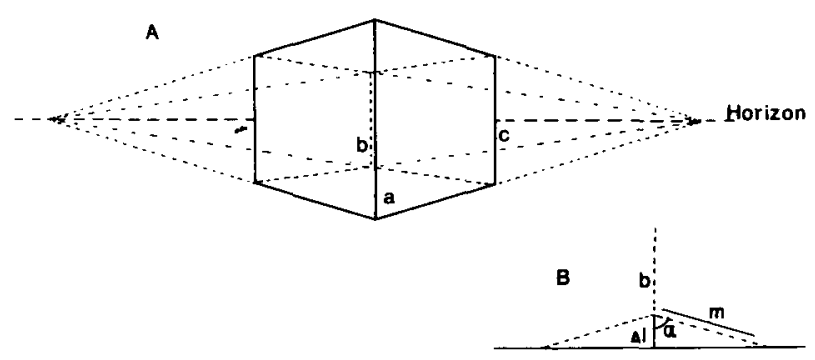

Figure 6. (A) The size-constancy operator tends to equalize the vertical edges of the cube. So a will be shortened while its position is closest to the observer and $b$ will be enlarged. (B) The enlargement of $b$ would be proportional to $\Delta l=m \cos \alpha$.

by depth cues can be described quite simply. In Figure 6 , a cube is pictured according to the simple rules of perspective. In the plane of the drawing, the center of gravity of the cube has to coincide with the horizon in order that the required symmetry of the MüllerLyer parts of the figure be obtained. The horizon is used for construction of the direction of the fins. In this construction, the side edges of the cube have to be situated at equal depth for obtaining equal fin angles of all fins. In this example, the size constancy operator would tend to enlarge the vertical edge in the back of the cube and to shorten the frontmost edge, both by an amount proportional to $\Delta \mathrm{l}=\mathrm{m} \cos \alpha$, with $\mathrm{m}=$ fin length and $\alpha=$ angle between shaft and fin, because this $\Delta \mathrm{l}$ restores all edges to equal length. This simple model predicts quantitatively the dependency on fin length, $m$, and fin angle, $\alpha$, in a straightforward way. To what extent this size constancy operator succeeds in controlling the length presumingly distorted by depth-producing factors remains to be seen, especially if the picture scenery is not completed with other depth cues. Ward et al. (1977) found weak, but consistent, evidence for the elicitation of depth by Müller-Lyer patterns.

Day (1972) convincingly stressed the fact that there may be more than one size induction mechanism operative. In the patterns under discussion, the mechanism of "size" induction of proximal elements" may become important. Müller-Lyer patterns with large fins produce shortening of shaft length irrespective of fin angle or fin lengths. With fin lengths exceeding a half shaft length, this effect becomes apparent.

We will restrict our analysis to fin lengths between one-half to one-fifth of the shaft length so that presumably we will not have to deal with this size induction operator.

\section{COMPARISON WITH EXPERIMENTAL DATA}

Davies and Spencer (1977) stress the experimental fact that, with fin angles of 30 to $60 \mathrm{deg}$ and with moderate fin lengths, the magnitude of the distortion of length is very accurately proportional to $\mathrm{m} \cos \alpha$.
The maximum distortion they report amounts to $20 \%$ of shaft length, with $\alpha=30 \mathrm{deg}$ and $m=1 / 4$ shaft length. Their figures were presented at a visual angle of about $10 \mathrm{deg}$, so their maximum distortion amounts to 2 deg of visual angle.

Restle and Decker (1977) report finding maximum illusions of about the same magnitude $(25 \%)$ in the older literature. They also report the interesting fact that there is a fin length-about one-third of the shaft length-that shows a maximum illusion. The maximum is dependent on fin angle and seems more pronounced with fins out. If, at each end of the shaft, one fin is left out on opposite sides, the maximum of the distortion drops considerably, from $25 \%$ to $10 \%$ of shaft length. In their experiments, this happens for fin angles between 30 and $60 \mathrm{deg}$ and, with their viewing distance, the maximum distortion of length is about $12 \mathrm{~min}$ of arc.

From these data it becomes apparent that maximum distortion of length may exceed $1 \mathrm{deg}$ of visual angle. This fact excludes both the filter theory and the combined filter and oriented bar detector model as the sole explanations of the illusion, because these mechanisms cannot explain effects larger than $15 \mathrm{~min}$ of arc, if only physiologically feasible parameters are considered. Moreover, the magnitude of the distortion in Figure 3 is not precisely proportional to $m \cos \alpha$ contrary to the accurate experimental data. The Davis and Spencer data (and also data of other authors cited by them) fit so closely to the $\mathrm{m} \cos \alpha$ relation that a correlation of .98 is obtained and errors never exceed $15 \%$. In comparing the distortion of Figure 3A, it must be concluded that the filter output shows a deviation from the $\mathrm{m} \cos \alpha$ proportion. This deviation from proportionality is not as devastating for the filter model as is the maximum obtainable illusion.

The Restle and Decker (1977) data for incomplete Müller-Lyer patterns show a much smaller illusion (12 min of arc). These figures do not appeal to a depth perception and therefore do not operate on the size constancy mechanism. Moreover, they found, for these incomplete patterns, a maximum illusion for fin lengths that were about .2-.3 fractions of the shaft lengths. As indicated by the right side of Figure 3, this would be expected if the filter model applied with $\sigma \cong 5 \mathrm{~min}$ of arc, which is a physiologically plausible value. Also in this figure, maxima are more pronounced for fins out, as in Restle and Decker's data.

Our conclusion is that, despite the fact that the filter properties of the peripheral visual system cannot play a main role in normal complete Müller-Lyer illusions, it nevertheless contributes significantly to the total effect.

As for the oriented bar detectors, we could not find experimental evidence for their role in the illusion of length. Indeed their physiological properties are not suitable to produce significant effects. In our opinion, Caelli's (1977) results with regard to the mis- 
apperception of the length of squiggles provide a better demonstration of the filter mechanism than of orientation detectors. Caelli's assumption that any orientation is measured as a ratio of vertical-tohorizontal extent does not find confirmation in electrophysiological experiments (Schiller et al., 1976).

The figural mechanism of the size constancy operator not only produces effects of sufficient magnitude, but also predicts the proportionality of the illusion with $\mathrm{m} \cos \alpha$ that is found in experiments. The size constancy operator in fact predicts illusions equal to $m \cos \alpha$. It is plausible, however, that this operator loses part of its effectiveness if the full depthproducing scenery is not present in the stimulus pattern. Judging from the experimental data, the size constancy operator is only $25 \%-50 \%$ effective here.

In older literature (Restle \& Decker, 1977), an optimum fin length is also found for complete MüllerLyer figures. In part this may be due to the visual filter, as explained above, but we must also expect, according to Day (1972), large proximal fins to have size reduction effects on the shaft. This effect may be larger for fins-out than for fins-in because large inward fins tend to cross. Our conclusion is that the size constancy operator is responsible for the major part of the illusion of length. If larger fin lengths are employed, figural explanations become rather complicated.

\section{DISCUSSION}

In the generator of Müller-Lyer illusions, at least two mechanisms are in effect. First, there exists a twodimensional filter in the peripheral visual system, as demonstrated by physiological and independent psychophysical measurements. This filter can be considered to be responsible for about one-fourth to onethird of the commonly measured distortions of length. It has the capability of predicting a fin length of maximum distortion, but it is less successful in predicting the experimental $\mathrm{m} \cos \alpha$ relation. Its most conspicuous and hardly investigated aspect is that its effect is heavily dependent on the viewing distance of the patterns.

The second successful mechanism is the sizeconstancy operator which restores sizes that are distorted by greater or lesser depth. This model predicts the $\mathrm{m} \cos \alpha$ relation directly. It does not predict, without further assumptions, a maximum effective fin length. The fact that the size-constancy operator does not show its full power for restoration of depthinduced distortions (being effective for only $50 \%$ or less) is paralleled by the study by Ward et al. (1977). They report that Müller-Lyer patterns show a consistent depth perception, but much less than would be predicted from laws of perspective.

Both models differ greatly in their predicted effects of dependency on viewing distance. In that regard it is remarkable that many investigators prefer to describe their stimulus patterns in measures of length when it would be advantageous for them to do so in visual angles. It might be possible that viewing distance is not important for the relative magnitude of the illusion as long as the pattern can be resolved by the eye, but such property is not self-evident. If it were true, it would mean that low-pass filtering or oriented bar detectors cannot give a significant contribution to the illusion effect. As explained before, much of the existing experimental data supported the conclusions that the peripheral visual filter played a minor role. In summary, the proposed mechanisms are more or less isolated in Figure 7: $a$ is the normal pattern; $b$ does not operate on depth analyzers or on oriented bar receptors; $c$ would not be influenced in the same way by filter properties because the light fins should contribute to the dark shaft in a reversed sense; $d$ would operate only on oriented line detectors.

Lester's (1977) suggestion that the size-constancy operator should also influence the thickness of the shaft does not apply, in our opinion, because this operator would need contours for identification of depth structures only and would operate on contours only. Line thickness cannot be considered a depth cue in this respect and does not elicit a size-constancy response.
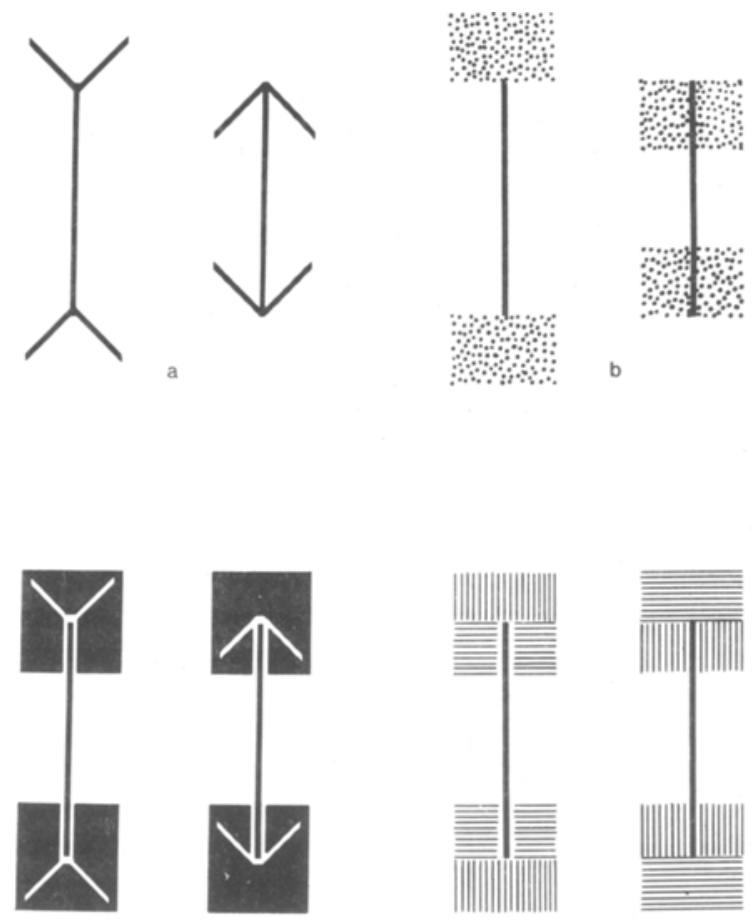

d

Figure 7. Different patterns may be devised which will operate on different mechanisms. (a) normal Müller-Lyer pattern; (b) no depth cues or orientation of lines; (c) conflicting filter output; and (d) exclusive operation on oriented line detectors. 
Pressey and Wilson (1977) pose a theoretical puzzle by observing that multiple fins do not show simple addition of effects or a simple averaging of effects. In our models, addition of distortion of length could never be expected. If the hydra-headed MüllerLyer pattern could elicit a depth at all, it would probably choose a mean depth interpretation. For inward double fins, such depth interpretation would be particularly difficult and that is where Pressey and Wilson find minimal illusions. A filter theory, on the other hand, would tend to predict an effect of a double pair of fins about equal to the fin pair with the larger effect.

In the present study, we have been comparing theories of two essentially different origins. On the one hand, we have investigated the influence of "hardware" properties of peripheral visual pathways, and, on the other hand, we have considered a "software" mechanism, as advocated by Gregory (1970), which can be used for analysis by the observer or not, depending on the available cues. Whether this last mechanism is employed totally unconsciously is not established. In any case, it seems wise to reckon with mechanisms which can be switched in or switched out under the influence of environmental depth cues.

\section{REFERENCES}

BARLOW, H. B. Reconstructing the visual image in space and time. Nature, 1979, 279, 189-190.

CaElli, T. Is perceived length affected by interactions between orientation detectors? Vision Research, 1977, 17, 837-841.

Davies, T. N., \& Spencer, J. An explanation for the MüllerLyer illusion. Perceptual and Motor Skills, 1977, 45, 219-224.

DAY, R. H. Visual spatial illusions: A general explanation. Science, 1972, 175, 1335-1340.

GaULD, A. A note on inappropriate constancy-scaling and the
Müller-Lyer illusion. British Journal of Psychology, 1975, 66, 307-309.

Gregony, R. L. The intelligent eye. London: Weidenfeld \& Nicolson, 1970.

Kawabata, N. Mathematical analysis of the visual illusion. IEEE Transactions on Systems, Man, and Cybernetics, 1976, SMC-6, 818-824.

LESTER, G. Size constancy scaling and the apparent thickness of the shaft in the Müller-Lyer illusion. Journal of General Psychology, 1977, 97, 307-308.

MülleR-Lyer, F. C. Zur Lehre von den optischen Tanschungen über Kontrast und Konfluxion. Zeitschrift für Psychologie, 1896, 9, 1-16; and 1896, 10, 421-431.

Pressey, A. W., \& Di Lollo, V. Effects of distance between standard and comparison lines on the Müller-Lyer illusion. Perception \& Psychophysics, 1978, 24, 415-419.

Pressey, A. W., \& Wilson, E. E. The hydra-headed MüllerLyer effect: A theoretical puzzle. Perception \& Psychophysics, 1977, 22, 392-394.

Restle, F., \& Decker, J. Size of the Müller-Lyer illusion as a function of its dimensions: Theory and data. Perception \& Psychophysics, 1977, 21, 489-503.

Schiller, P. H., Finlay, B. L., \& Volman, S. F. Quantitative studies of single-cell properties in monkey striate cortex. II. Orientation specificity and ocular dominance. Journal of Neurophysiology, 1976, 39, 1320-1333.

Sмiтh, D. A. A descriptive model for perception of optical illusions. Journal of Mathematical Psychology, 1978, 17, 64-85.

Ward, L. M, Porac, C., Coren, S., \& Gingus, J. S. The case of misapplied constancy scaling: Depth associations elicited by illusion configurations. American Journal of Psychology, 1977, 90, 609-620.

Warren, R. M., \& Bachford, J. A. Müller-Lyer illusions: Their origin in processes facilitating object recognition. Perception, 1977, 6, 615-626.

Wilson, H. R., \& Bergen, J. R. A four mechanism model for threshold spatial vision. Vision Research, 1979, 19, 19-32.

Yarbus, A. L. Eye movements and vision. New York: Plenum Press, 1967.

(Received for publication May 30, 1980; revision accepted January 30,1981 .) 